\title{
Quadratic third-order tensor optimization problem with quadratic constraints
}

\author{
Lixing Yang ${ }^{1}$, Qingzhi Yang ${ }^{2}$ and Xiaoming Zhao ${ }^{2, *}$ \\ ${ }^{1}$ Department of Electrical and Computer Engineering, New Jersey Institute of Technology, USA, \\ ${ }^{2}$ School of Mathematical Sciences and LPMC, Nankai University, P.R.China.
}

Received 23 January 2014; Accepted 10 April 2014

Editor: Zhenghai Huang

\begin{abstract}
Quadratically constrained quadratic programs (QQPs) problems play an important modeling role in many diverse problems. These problems are in general NP hard and numerically intractable. Semidefinite programming (SDP) relaxations often provide good approximate solutions to these hard problems. For several special cases of QQP, e.g., convex programs and trust region subproblems, SDP relaxation provides the exact optimal value, i.e., there is a zero duality gap. However, this is not true for the general QQP, or even the QQP with two convex constraints, but a nonconvex objective. In this paper, we consider a certain QQP where the variable is neither vector nor matrix but a third-order tensor. This problem can be viewed as a generalization of the ordinary QQP with vector or matrix as it's variable. Under some mild conditions, we first show that SDP relaxation provides exact optimal solutions for the original problem. Then we focus on two classes of homogeneous quadratic tensor programming problems which have no requirements on the constraints number. For one, we provide an easily implemental polynomial time algorithm to approximately solve the problem and discuss the approximation ratio. For the other, we show there is no gap between the SDP relaxation and itself.
\end{abstract}

Keywords quadratic third-order tensor programming, semidefinite relaxation, SDP relaxation, approximate algorithm

$\operatorname{MSC}(\mathbf{2 0 0 0}): 15 \mathrm{~A} 69,90 \mathrm{C} 20,90 \mathrm{C} 26,90 \mathrm{C} 59$

DOI: $10.19139 /$ soic.v2i2.67

${ }^{*}$ Correspondence to: School of Mathematical Sciences and LPMC, Nankai University, P.R.China.

ISSN 2310-5070 (online) ISSN 2311-004X (print)

Copyright (c) 2014 International Academic Press 


\section{Introduction}

A tensor is a multidimensional array. More formally, an $N$-way or $N$ th-order tensor is an element of the tensor product of $N$ vector spaces, each of which has its own coordinate system. A first-order tensor is a vector, a second-order tensor is a matrix, a third-order tensor has three indices and tensors of order three or higher are called higher-order tensors. Tensors have been widely utilized in Electrical Engineering since the nineties [16], and in particular in Antenna Array Processing [4] or Telecommunications [9] [13].

In this paper, we mainly consider the following nonconvex quadratic optimization problem of the form

$$
\begin{aligned}
\min f_{0}(\mathcal{X}) & =\sum_{i, j=1}^{n_{1}} \sum_{k=1}^{n_{2}} \sum_{l=1}^{n_{3}} A_{i j}^{0} \mathcal{X}_{i k l} \mathcal{X}_{j k l}+2 \sum_{i=1}^{n_{1}} \sum_{k=1}^{n_{2}} \sum_{l=1}^{n_{3}} B_{i k}^{0} \mathcal{X}_{i k l}+c^{0} \\
\text { s.t. } f_{p}(\mathcal{X})= & \sum_{i, j=1}^{n_{1}} \sum_{k=1}^{n_{2}} \sum_{l=1}^{n_{3}} A_{i j}^{p} \mathcal{X}_{i k l} \mathcal{X}_{j k l}+2 \sum_{i=1}^{n_{1}} \sum_{k=1}^{n_{2}} \sum_{l=1}^{n_{3}} B_{i k}^{p} \mathcal{X}_{i k l}+c^{p} \leq 0 \\
& p=1, \cdots, m
\end{aligned}
$$

where $A^{p}=\left(A^{p}\right)^{T} \in \mathbb{R}^{n_{1} \times n_{1}}, B^{p} \in \mathbb{R}^{n_{1} \times n_{2}}, c^{p} \in \mathbb{R}, p=0,1, \cdots, m$ and $\mathcal{X} \in$ $\mathbb{R}^{n_{1} \times n_{2} \times n_{3}}$ is a third-order tensor. Problem of the above type is actually an extension of the quadratic matrix programming (QMP) problem [6, 2] and quadratically constrained quadratic programs (QQPs) $[5,15,3]$ if we let $n_{3}=1$ and $n_{2}, n_{3}=1$ respectively. We call problem (1.1) a quadratic third-order tensor programming (QTTP) problem.

Tight semidefinite programming (SDP) relaxation is known to hold for only a few classes of nonconvex QQPs such as trust region problem [5]. Many extensions of this problem were considered in $[12,15]$ while these results cannot be extended to QQPs involving two constraints [17]. As a generalization of QQPs with vector variables, Beck [2] proved stronger results to QMP problems. Now after extending "matrix" to the "third-order tensor" situation, we will establish the same tight SDP relaxation results for QTTP problems with at most $n_{2}$ constraints (see section 2). However if QTTP problem (1.1) is homogeneous, still we can get the tightness result in spite of the constraints number under some conditions (see section 4).

Tensors can be multiplied together, though obviously the notation and symbols for this are much more complex than for matrices. For a full treatment of tensor multiplication see, e.g., Bader and Kolda [1]. In [8], they consider the tensor $n$ mode product, i.e., multiplying a tensor by a matrix (or a vector) in mode $n$ :

$$
\left(\mathcal{X} \times{ }_{n} U\right)_{i_{1} \cdots i_{n-1} j i_{n+1} \cdots N}=\sum_{i_{n}=1}^{I_{n}} \mathcal{X}_{i_{1} \cdots i_{N}} U_{j i_{n}},
$$


here tensor $\mathcal{X} \in \mathbb{R}^{I_{1} \times \cdots \times I_{N}}$ and matrix $U \in \mathbb{R}^{J \times I_{n}}$. Using this definition, we seek to find a third-order tensor $\mathcal{X}$ which solves the following optimization problem:

$$
\min \left\|\mathcal{X} \times{ }_{1} A-\mathcal{B}\right\|^{2}
$$

where $A \in \mathbb{R}^{n_{1} \times n_{1}}$ and $\mathcal{X}, \mathcal{B} \in \mathbb{R}^{n_{1} \times n_{2} \times n_{3}}$ are two third-order tensors. The norm of a tensor $\mathcal{X}$ is the square root of the sum of the squares of all its elements, i.e.,

$$
\|\mathcal{X}\|=\sqrt{\sum_{i=1}^{n_{1}} \sum_{j=1}^{n_{2}} \sum_{k=1}^{n_{3}} \mathcal{X}_{i j k}^{2}} .
$$

This is analogous to the matrix Frobenius norm, which is denoted $\|A\|$ for a matrix $A$. If tensor $\mathcal{B}$ satisfies $\mathcal{B}_{i j k}=B_{i j}$ for $1 \leq k \leq n_{3}$, problem (1.2) can be rewritten as a nonconstraint QTTP problem:

$$
\begin{aligned}
\min & \sum_{i, j=1}^{n_{1}} \sum_{k=1}^{n_{2}} \sum_{l=1}^{n_{3}}\left(A^{T} A\right)_{i j} \mathcal{X}_{i k l} \mathcal{X}_{j k l}-2 \sum_{i=1}^{n_{1}} \sum_{k=1}^{n_{2}} \sum_{l=1}^{n_{3}}\left(\sum_{s=1}^{n_{1}} A_{s i} B_{s k}\right) \mathcal{X}_{i k l} \\
& +n_{3} \sum_{i=1}^{n_{1}} \sum_{k=1}^{n_{2}} B_{i k}^{2} .
\end{aligned}
$$

The remaining of the paper is organized as follows: In section 2, we homogenize the QTTP problem and change it to a separable QMP problem and show that the optimal values can be obtained exactly by its SDP relaxation under some mild conditions. In section 3, we concentrate on the homogeneous QTTP problem. Although it is still NP-hard, we can approximately solve it in polynomial time. In section 4, we present a special class of homogeneous QTTP problems with nonpositive off-diagonal elements and show that their optimal values can be obtained exactly by SDP relaxations and more precisely be solved without gap.

Throughout this paper, we use the following notations: Vectors are denoted by lowercase letters, e.g., $x$, matrices by uppercase letters, e.g., $X$, and third-order tensors by calligraphic capitals, e.g., $\mathcal{X}$. Let $x_{i}, X_{i j}$ and $\mathcal{X}_{i j k}$ denote the $i$ th, $i j$ th and $i j k$ th component of $x, X$ and $\mathcal{X}$ respectively. For matrix $A$ and $B, A \succeq 0$ means that $A$ is a positive semidefinite matrix, $\operatorname{rank}(A)$ denotes the rank of $A$, $\operatorname{Tr} A$ denotes the trace of $A, A^{T}$ denotes the transpose matrix of $A$, and $A \bullet B$ stands for the standard matrix inner product, i.e., $A \bullet B=\operatorname{Tr} A^{T} B$. For random variable $\xi, \mathbf{E} \xi$ denotes the expectation of $\xi . \mathbb{S}^{n}\left(\mathbb{S}_{+}^{n}\right)$ is the set of all real symmetric $n \times n$ matrices(positive semidefinite matrices). $0_{n_{1} \times n_{2}}$ is the $n_{1} \times n_{2}$ matrix of zeros, $I_{r}$ is the $r \times r$ identity matrix, and $E_{s t}^{r}$ is the $r \times r$ matrix with 1 at the st th component and 0 elsewhere. The value of the optimal objective function of an optimization problem

$$
\text { (P) } \min \{f(x): x \in C\}
$$

is denoted by $v_{\min }(P)$. 


\section{Semidefinite relaxations of the QTTP problem and tightness results}

We construct a similar scheme provided by A. Beck [2] to obtain a SDP relaxation for (QTTP). By solving the relaxed problem, in this section, we will show that under some mild conditions, tightness of (QTTP) is hold with at most $n_{2}$ constraints.

As the method used to homogenize a quadratic vector function, we consider the following homogenization procedure to QTT function: Let

$$
f(\mathcal{X})=\sum_{i, j=1}^{n_{1}} \sum_{k=1}^{n_{2}} \sum_{l=1}^{n_{3}} A_{i j} \mathcal{X}_{i k l} \mathcal{X}_{j k l}+2 \sum_{i=1}^{n_{1}} \sum_{k=1}^{n_{2}} \sum_{l=1}^{n_{3}} B_{i k} \mathcal{X}_{i k l}+c
$$

like (QTTP). The homogenized QTT function is denoted by $f^{H}$ : $\mathbb{R}^{\left(n_{1}+n_{2}\right) \times n_{2} \times n_{3}} \rightarrow \mathbb{R}$ and given by

$$
\begin{aligned}
f^{H}(\mathcal{X} ; \mathcal{Z}) & =\sum_{i, j=1}^{n_{1}} \sum_{k=1}^{n_{2}} \sum_{l=1}^{n_{3}} A_{i j} \mathcal{X}_{i k l} \mathcal{X}_{j k l}+2 \sum_{i=1}^{n_{1}} \sum_{s, k=1}^{n_{2}} \sum_{l=1}^{n_{3}} B_{i s} \mathcal{X}_{i k l} \mathcal{Z}_{s k l} \\
& +\frac{c}{n_{2} n_{3}} \sum_{s, k=1}^{n_{2}} \sum_{l=1}^{n_{3}} \mathcal{Z}_{s k l}^{2} .
\end{aligned}
$$

The homogenous function $f^{H}$ satisfies the following easily verifiable properties, which will become useful in what follows

\section{Property 2.1}

Let matrices $\left\{Z^{l}\right\}_{l=1}^{n_{3}} \in \mathbb{R}^{n_{2} \times n_{2}}$ satisfy

$$
Z_{s k}^{l}=\mathcal{Z}_{s k l}
$$

for $s, k=1, \cdots, n_{2}$ and $l=1, \cdots, n_{3}$.

(1). If $Z^{1}, \cdots, Z^{n_{3}}=I_{n_{2}}$, we call $\mathcal{Z} \in \mathbb{R}^{n_{2} \times n_{2} \times n_{3}}$ the identity three order tensor and denote it by $\mathcal{I}$, then $f(\mathcal{X})=f^{H}(\mathcal{X}, \mathcal{I})$;

(2). If $Z^{1}\left(Z^{1}\right)^{T}, \cdots, Z^{n_{3}}\left(Z^{n_{3}}\right)^{T}=I_{n_{2}}$, set

$$
\mathcal{D}_{i s l}=\sum_{k=1}^{n_{2}} \mathcal{X}_{i k l} \mathcal{Z}_{s k l}
$$

for $i=1, \cdots, n_{1}, s=1, \cdots, n_{2}$ and $l=1, \cdots, n_{3}$, then $f(\mathcal{D})=f^{H}(\mathcal{X} ; \mathcal{Z})$.

Using the above homogenization procedure for QTT functions, we are able to prove (see Proposition 2.1 below) that (QTTP) is equivalent to some homogeneous QTTP problem. 
Proposition 2.1

Consider the following homogenized version of (QTTP):

$$
\begin{aligned}
(H Q T P) \min & f_{0}^{H}(\mathcal{X} ; \mathcal{Z}) \\
\text { s.t. } \quad & f_{p}^{H}(\mathcal{X} ; \mathcal{Z}) \leq 0, \quad p=1, \cdots, m \\
& g_{s t l}(\mathcal{X} ; \mathcal{Z})=\delta_{s t}, \quad 1 \leq s \leq t \leq n_{2}, l=1, \cdots, n_{3}, \\
& \mathcal{X} \in \mathbb{R}^{n_{1} \times n_{2} \times n_{3}}, \mathcal{Z} \in \mathbb{R}^{n_{2} \times n_{2} \times n_{3}}
\end{aligned}
$$

where $g_{s t l}=\sum_{k} \mathcal{Z}_{s k l} \mathcal{Z}_{t k l}$ and $\delta_{s t}$ is the Kronecker delta, then we have:

1. If (QTTP) is solvable and $\mathcal{X}^{*}$ is an optimal solution, then (HQTP) is solvable and order tensor

$\left(\mathcal{X}^{*} ; \mathcal{I}\right)$ is an optimal solution, where $\mathcal{I} \in \mathbb{R}^{n_{2} \times n_{2} \times n_{3}}$ is the identity three defined in property 2.1 .

2. If (HQTP) is solvable and $\left(\mathcal{X}^{*} ; \mathcal{Z}^{*}\right)$ is an optimal solution, then (QTTP) is solvable

and $\mathcal{D}^{*}$ is an optimal solution, where $\mathcal{D}^{*}$ is defined as (2.2).

Proof. Through property 2.1 , we note that $f(\mathcal{D})=f^{H}(\mathcal{X} ; \mathcal{Z})$ for every feasible solution $(\mathcal{X} ; \mathcal{Z})$ of (HQTP), since the system of equalities

$$
g_{s t l}(\mathcal{X} ; \mathcal{Z})=\delta_{s t}, \quad 1 \leq s \leq t \leq n_{2}, l=1, \cdots, n_{3}
$$

is actually equivalent to $Z^{1}\left(Z^{1}\right)^{T}, \cdots, Z^{n_{3}}\left(Z^{n_{3}}\right)^{T}=I_{n_{2}}$.

1 . For every feasible solution $(\mathcal{X} ; \mathcal{Z})$ of (HQTP), combined with property 2.1 we have

$$
f_{0}^{H}(\mathcal{X} ; \mathcal{Z})=f_{0}(\mathcal{D}), \quad f_{0}^{H}\left(\mathcal{X}^{*} ; \mathcal{I}\right)=f_{0}\left(\mathcal{X}^{*}\right)
$$

Meanwhile

$$
f_{p}(\mathcal{D})=f_{p}^{H}(\mathcal{X} ; \mathcal{Z}) \leq 0, \quad p=1, \cdots, m
$$

shows that $\mathcal{D}$ is a feasible solution of (QTTP), which means $f_{0}(\mathcal{D}) \geq f_{0}\left(\mathcal{X}^{*}\right)$. From the above equalities, we argue that $\left(\mathcal{X}^{*} ; \mathcal{I}\right)$ is an optimal solution of (HQTP).

2. Similar to the above method, for every $\mathcal{X}$ in the feasible set of (QTTP), we have

$$
f_{0}(\mathcal{X})=f_{0}^{H}(\mathcal{X} ; \mathcal{I}) \geq f_{0}^{H}\left(\mathcal{X}^{*} ; \mathcal{Z}^{*}\right)=f_{0}\left(\mathcal{D}^{*}\right),
$$

which shows that $\mathcal{D}^{*}$ is an optimal solution of (QTTP).

Notice that (QTTP) and (HQTP) have the same value of the optimal objective function via the proof of Proposition 2.1, i.e.

$$
v_{m i n}(Q T T P)=v_{\min }(H Q T P)
$$


So they are actually equivalent optimization problem. From now on, we will only consider (HQTP) instead of (QTTP).

For QTT function (2.1), let matrix sequences $\left\{Z^{l}\right\}_{l=1}^{n_{3}}$ and $\left\{X^{l}\right\}_{l=1}^{n_{3}}$ satisfy

$$
Z_{s k}^{l}=\mathcal{Z}_{s k l}, \quad X_{i k}^{l}=\mathcal{X}_{i k l}
$$

with $i=1, \cdots, n_{1}, s, k=1, \cdots, n_{2}$ and $l=1, \cdots, n_{3}$. For convenience, we adopt the following matrix notation:

$$
M_{f}=\left(\begin{array}{cc}
A & B \\
B^{T} & \frac{c}{n_{2} n_{3}} I_{n_{2}}
\end{array}\right) \quad \text { and } \quad W^{l}=\left(\begin{array}{c}
X^{l} \\
Z^{l}
\end{array}\right) .
$$

Then we can rewrite $f^{H}(\mathcal{X} ; \mathcal{Z})$ as

$$
f^{H}(\mathcal{X} ; \mathcal{Z})=\sum_{l=1}^{n_{3}} M_{f} \bullet W^{l}\left(W^{l}\right)^{T} .
$$

It holds that (HQTP) is equivalent to

$$
\begin{array}{ll}
\min & \sum_{l=1}^{n_{3}} M_{f_{0}} \bullet W^{l}\left(W^{l}\right)^{T} \\
\text { s.t. } & \sum_{l=1}^{n_{3}} M_{f_{p}} \bullet W^{l}\left(W^{l}\right)^{T} \leq 0, \quad p=1, \cdots, m, \\
& N^{s t} \bullet W^{l}\left(W^{l}\right)^{T}=2 \delta_{s t}, \quad 1 \leq s \leq t \leq n_{2}, \\
& W^{l} \in \mathbb{R}^{\left(n_{1}+n_{2}\right) \times n_{2}}, \quad l=1, \cdots, n_{3}
\end{array}
$$

with

$$
N^{s t}=\left(\begin{array}{cc}
0_{n_{1} \times n_{1}} & 0_{n_{1} \times n_{2}} \\
0_{n_{2} \times n_{1}} & E_{s t}^{n_{2}}+E_{t s}^{n_{2}}
\end{array}\right), \quad 1 \leq s \leq t \leq n_{2} .
$$

Now after some constructions, (QTTP) has been transferred to problem (2.4), where the objective function is in the form of the sum of $n_{3}$ individual functions without crossed variables. In fact, problem (2.4) can be viewed as a special kind of separable QMP problems. Based on this characterization, making use of the rank reduction algorithm of [11], we can deduce the tightness between (QTTP) and its SDP relaxation.

Let $U^{l}=W^{l}\left(W^{l}\right)^{T}$ for $l=1, \cdots, n_{3}$, which can be equivalently written as

$$
\left\{\begin{array}{l}
U^{l} \in \mathbb{S}_{+}^{n_{1}+n_{2}} \\
\operatorname{rank}\left(U^{l}\right) \leq n_{2}
\end{array}\right.
$$

Stat., Optim. Inf. Comput. Vol. 2, June 2014. 
Omitting constraints $\left\{\operatorname{rank}\left(U^{l}\right) \leq n_{2}\right\}_{l=1}^{n_{3}}$, we finally arrive at the SDP relaxation of (HQTP):

$$
\begin{aligned}
(\text { RHQTP }) \min & \sum_{l=1}^{n_{3}} M_{f_{0}} \bullet U^{l} \\
\text { s.t. } & \sum_{l=1}^{n_{3}} M_{f_{p}} \bullet U^{l} \leq 0, \quad p=1, \cdots, m, \\
& N^{s t} \bullet U^{l}=2 \delta_{s t}, \quad 1 \leq s \leq t \leq n_{2}, \\
& U^{l} \in \mathbb{S}_{+}^{n_{1}+n_{2}}, \quad l=1, \cdots, n_{3},
\end{aligned}
$$

The SDP problem (RHQTP) has a dual, which is given by

$$
\begin{aligned}
(D H Q T P) \quad \max & -\sum_{l=1}^{n_{3}} \operatorname{Tr} \Psi^{l} \\
\text { s.t. } \quad & V^{l}=M_{f_{0}}+\sum_{p=1}^{m} \lambda_{p} M_{f_{p}}+\left(\begin{array}{cc}
0_{n_{1} \times n_{1}} & 0_{n_{1} \times n_{2}} \\
0_{n_{2} \times n_{1}} & \Psi^{l}
\end{array}\right), \\
& V^{l} \succeq 0, \quad \lambda_{p} \geq 0, \\
& \Psi^{l} \in \mathbb{S}^{n_{2}}, \quad l=1, \cdots, n_{3}, p=1, \cdots, m .
\end{aligned}
$$

For the sake of completeness, we present the constructive rank reduction procedure [2, 11] again (Proposition 2.2 below) to reach a rank constraint of the optimal solution of (RHQTP).

\section{Proposition 2.2}

Suppose that (RHQTP) and its dual (DHQTP) are solvable. Then, (RHQTP) has always an optimal solution $\left(U^{* l}\right)_{l=1}^{n_{3}}$ such that

$$
\sum_{l=1}^{n_{3}} \frac{\operatorname{rank}\left(U^{* l}\right)\left(\operatorname{rank}\left(U^{* l}\right)+1\right)}{2} \leq m+\frac{n_{2} n_{3}\left(n_{2}+1\right)}{2} .
$$

Proof. Suppose $\left(U^{l}\right)_{l=1}^{n_{3}}$ and $\left(\left(V^{l}\right)_{l=1}^{n_{3}},\left(\lambda_{p}\right)_{p=1}^{m},\left(\Psi^{l}\right)_{l=1}^{n_{3}}\right)$ are optimal solutions of (RHQTP) and (DHQTP), respectively (existence can be ensured by assuming that both the primal and dual SDPs have interior points in their feasible regions, respectively). Notice that they comply with the complementary conditions

$$
\lambda_{p} \sum_{l} M_{f_{p}} \bullet U^{l}=0 \quad \text { for } p=1, \cdots, m,
$$

and

$$
U^{l} V^{l}=0 \quad \text { for } l=1, \cdots, n_{3} .
$$


Let $R_{l}=\operatorname{rank}\left(U^{l}\right), \quad l=1, \cdots, n_{3}$. By decomposing $U^{l}=Y^{l}\left(Y^{l}\right)^{T}, \quad Y^{l} \in$ $\mathbb{R}^{\left(n_{1}+n_{2}\right) \times R_{l}}$, it holds that

$$
\begin{array}{r}
\sum_{l} M_{f_{P}} \bullet U^{l}=\sum_{l}\left(Y^{l}\right)^{T} M_{f_{p}} Y^{l} \bullet I_{R_{l}} \leq 0 \quad p=1, \cdots, m, \\
N^{s t} \bullet U^{l}=\left(Y^{l}\right)^{T} N^{s t} Y^{l} \bullet I_{R_{l}}=2 \delta_{s t} \quad 1 \leq s \leq t \leq n_{2}, l=1, \cdots, n_{3} .
\end{array}
$$

Consider the following system of linear equations:

$$
\begin{array}{r}
\sum_{l}\left(Y^{l}\right)^{T} M_{f_{p}} Y^{l} \bullet \Delta^{l}=0 \quad p=1, \cdots, m, \\
\left(Y^{l}\right)^{T} N^{s t} Y^{l} \bullet \Delta^{l}=0 \quad 1 \leq s \leq t \leq n_{2}, l=1, \cdots, n_{3}
\end{array}
$$

with $\Delta^{l} \in \mathbb{S}^{R_{l}}$ for $l=1, \cdots, n_{3}$. Note that there are $\frac{R_{l}\left(R_{l}+1\right)}{2}$ unknowns in the entries of $\Delta^{l}$; therefore the system (2.7) has $m+\frac{n_{3} n_{2}\left(n_{2}+1\right)}{2}$ equations and $\sum_{l} \frac{R_{l}\left(R_{l}+1\right)}{2}$ unknowns. If $\sum_{l} \frac{R_{l}\left(R_{l}+1\right)}{2} \geq m+\frac{n_{3} n_{2}\left(n_{2}+1\right)}{2}$, then there is a nonzero solution of the system of linear (2.7), say, $\left(\Delta^{l}\right)_{l=1}^{n_{3}}$. Let $\sigma_{l k}$ with $k=$ $1, \cdots, R_{l}$ be eigenvalues of $\Delta^{l}$ for $l=1, \cdots, n_{3}$. Let $l_{0}$ and $k_{0}$ be such that

$$
\left|\sigma_{l_{0} k_{0}}\right|=\max \left\{\left|\sigma_{l k}\right|: 1 \leq k \leq R_{l}, 1 \leq l \leq n_{3}\right\} .
$$

Thus it is easily seen that the matrices

$$
I_{R_{l}}-\frac{1}{\sigma_{l_{0} k_{0}}} \Delta^{l} \succeq 0 \quad l=1, \cdots, n_{3} .
$$

Let $\bar{U}^{l}=Y^{l}\left(I_{R_{l}}-\frac{1}{\sigma_{l_{0} k_{0}}} \Delta^{l}\right)\left(Y^{l}\right)^{T}$ for $l=1, \cdots, n_{3}$, clearly $\bar{U}^{l} \succeq 0$. Noticing that

$$
\sum_{l} M_{f_{p}} \bullet \bar{U}^{l} \leq 0, \quad N^{s t} \bullet \bar{U}^{l}=2 \delta_{s t}
$$

with $p=1, \cdots, m, 1 \leq s \leq t \leq n_{2}$ and $l=1, \cdots, n_{3}$, it follows that $\left(\bar{U}^{l}\right)_{l=1}^{n_{3}}$ is a feasible solution of (RHQTP). Meanwhile, it is easy to prove that (2.5) (2.6) and (2.7), together with $\bar{U}^{l}, V^{l} \succeq 0, l=1, \cdots, n_{3}$, gives that

$$
\lambda_{p} \sum_{l} M_{f_{p}} \bullet \bar{U}^{l}=0, \quad \bar{U}^{l} V^{l}=0 \quad \text { for } p=1, \cdots, m, l=1, \cdots, n_{3} .
$$

Therefore the complementary conditions are satisfied, which means $\left(\bar{U}^{l}\right)_{l=1}^{n_{3}}$ is still an optimal solution of (RHQTP). Note that $\sum_{l} \operatorname{rank}\left(\bar{U}^{l}\right) \leq \sum_{l} R_{l}-1$, i.e. sum of the ranks of the optimal solutions reduce at least one. Repeat the above rank-deduction procedure, finally we will get one optimal solution $\left(U^{* l}\right)_{l=1}^{n_{3}}$ of (RHQTP) such that

$$
\sum_{l=1}^{n_{3}} \frac{\operatorname{rank}\left(U^{* l}\right)\left(\operatorname{rank}\left(U^{* l}\right)+1\right)}{2} \leq m+\frac{n_{2} n_{3}\left(n_{2}+1\right)}{2},
$$


which completes the proof.

Using Proposition 2.2, we are now able to show that (QTTP) with at most $n_{2}$ constraints possess a tight SDP relaxation under some mild conditions.

Theorem 2.1

Suppose that (RHQTP) and its dual (DHQTP) are solvable. If the rank of each optimal solutions of (RHQTP) is no less than $n_{2}$ and $m \leq n_{2}$, then, (QTTP) is solvable and $v_{\min }(Q T T P)=v_{\min }(R H Q T P)$.

Proof. By assumption, any given optimal solution $\left(U^{l}\right)_{l=1}^{n_{3}}$ of (RHQTP) satisfies $\operatorname{rank}\left(U^{l}\right) \geq n_{2}$ for $l=1, \cdots, n_{3}$. It follows by Proposition 2.2 that (RHQTP) has an optimal solution $\left(U^{* l}\right)_{l=1}^{n_{3}}$ such that

$$
\frac{n_{2} n_{3}\left(n_{2}+1\right)}{2} \leq \sum_{l=1}^{n_{3}} \frac{\operatorname{rank}\left(U^{* l}\right)\left(\operatorname{rank}\left(U^{* l}\right)+1\right)}{2} \leq n_{2}+\frac{n_{2} n_{3}\left(n_{2}+1\right)}{2} .
$$

This implies $\operatorname{rank}\left(U^{* l}\right)=n_{2}$ for $l=1, \cdots, n_{3}$. As a result, (RHQTP) is equivalent to problem (2.4). Hence by (2.3), we have

$$
v_{\text {min }}(Q T T P)=v_{\text {min }}(H Q T P)=v_{\text {min }}(2.4)=v_{\text {min }}(R H Q T P),
$$

which completes the proof.

\section{Approximation algorithms for the homogeneous QTTP problem}

The focus of this section, in particular, is on nonconvex QTTPs which involve positive semidefinite matrices in constraints, and the objective function and constrains both are homogeneous. Through a simple randomized polynomial-time procedure proposed by AMC So et al [14], we can extract a feasible solution of (QTTP) from the optimal solution of its SDP relaxation. And the approximation ratio is $\Omega\left(\frac{1}{\log m}\right)$ or $\Omega\left(\frac{1}{\sqrt{\log m}}\right)$ which depends on the magnitude of $n_{2}$.

Consider the following optimization problems:

$$
\begin{aligned}
(H Q-1) \quad v_{\text {minqp }}^{*}= & \min \sum_{i, j=1}^{n_{1}} \sum_{k=1}^{n_{2}} \sum_{l=1}^{n_{3}} A_{i j}^{0} \mathcal{X}_{i k l} \mathcal{X}_{j k l} \\
\text { s.t. } \quad & \sum_{i, j=1}^{n_{1}} \sum_{k=1}^{n_{2}} \sum_{l=1}^{n_{3}} A_{i j}^{p} \mathcal{X}_{i k l} \mathcal{X}_{j k l} \leq \alpha^{p}, \\
& A^{p} \succeq 0, \quad p=1, \cdots, m,
\end{aligned}
$$

where $A^{0}, A^{p} \in \mathbb{S}^{n_{1}}$ and $\alpha^{p} \in \mathbb{R}$ for $p=1, \cdots, m$. we can see that (HQ-1) is (QTTP)'s simplification by omitting the monomial term and the constant term. 
Unfortunately, (HQ-1) itself is still NP-hard which has been proved in [10] as $n_{2}=$ $n_{3}=1$. However, it is possible to approximately solve the problem by deriving a feasible solution from its SDP relaxation. And we see that the approximating procedure can be completed in polynomial time.

Similar to the argument in section 2, we can rewrite (HQ-1) with another equivalent form:

$$
\begin{aligned}
& v_{\text {minqp }}^{*}=\min \sum_{l=1}^{n_{3}} A^{0} \bullet U^{l} \\
& \text { s.t. } \quad \sum_{l=1}^{n_{3}} A^{p} \bullet U^{l} \leq \alpha^{p}, \quad p=1, \cdots, m, \\
& U^{l} \succeq 0, \quad \operatorname{rank}\left(U^{l}\right) \leq n_{2},
\end{aligned}
$$

where $U^{1}, \cdots, U^{m} \in \mathbb{S}^{n_{1}}$, and its natural SDP relaxation is given by:

$$
\begin{aligned}
& v_{\text {minsdp }}^{*}=\min \sum_{l=1}^{n_{3}} A^{0} \bullet U^{l} \\
& \text { s.t. } \quad \sum_{l=1}^{n_{3}} A^{p} \bullet U^{l} \leq \alpha^{p}, \\
& U^{l} \succeq 0, \quad p=1, \cdots, m .
\end{aligned}
$$

Now we introduce a low-rank approximate solution to a system of linear equations in symmetric positive semidefinite matrices, which will be used to derive an approximate solution to our homogeneous QTTP problem (HQ-1).

\section{Lemma 3.1}

[14] Let $A_{1}, \cdots, A_{m} \in \mathbb{R}^{n \times n}$ be symmetric positive semidefinite matrices, where $n<\sqrt{2 m}$. Then, for any $d \geq 1$, there exists an $X_{0} \succeq 0$ with $\operatorname{rank}\left(X_{0}\right) \leq d$ such that:

$$
\beta(m, n, d) \cdot \operatorname{Tr} A_{i} \leq A_{i} \bullet X_{0} \leq \alpha(m, n, d) \cdot \operatorname{Tr} A_{i} \quad \text { for } i=1, \cdots, m,
$$

where:

$$
\alpha(m, n, d)= \begin{cases}1+\frac{12 \log (4 m r)}{d} & \text { for } 1 \leq d \leq 12 \log (4 m r) \\ 1+\sqrt{\frac{12 \log (4 m r)}{d}} & \text { for } d>12 \log (4 m r)\end{cases}
$$

and

$$
\beta(m, n, d)= \begin{cases}\frac{1}{e(2 m)^{2 / d}} & \text { for } 1 \leq d \leq 4 \log (2 m) \\ \max \left\{\frac{1}{e(2 m)^{2 / d}}, 1-\sqrt{\frac{4 \log (2 m)}{d}}\right\} & \text { for } d>4 \log (2 m)\end{cases}
$$

Moreover, there exists an efficient randomized algorithm for finding such an $X_{0}$. 
Our main result in this section is the following:

\section{Theorem 3.1}

Let $\left(U^{* l}\right)_{l=1}^{n_{3}}$ be an optimal solution to (3.2). We can extract a feasible solution $\mathcal{X}_{0}$ of (HQ-1) from $\left(U^{* l}\right)_{l=1}^{n_{3}}$ such that

$$
v_{\text {minqp }}^{*} \leq \sum_{i j k l} A_{i j}^{0} \mathcal{X}_{i k l}^{0} \mathcal{X}_{i k l}^{0} \leq \frac{1}{\alpha\left(m, n_{1}, n_{2}\right)} v_{\text {minqp }}^{*}
$$

where:

$$
\alpha\left(m, n_{1}, n_{2}\right)= \begin{cases}1+\frac{12 \log (4 m h)}{n_{2}} & \text { for } 1 \leq n_{2} \leq 12 \log (4 m h) \\ 1+\sqrt{\frac{12 \log (4 m h)}{n_{2}}} & \text { for } n_{2}>12 \log (4 m h)\end{cases}
$$

and $h=\min \left\{\sqrt{2 m}, n_{1}\right\}$.

Proof. Using Proposition 2.2, we know there exists an optimal solution $\left(U^{* l}\right)_{l=1}^{n_{3}}$ of problem (3.2) such that

$$
\sum_{l=1}^{n_{3}} \frac{\operatorname{rank}\left(U^{* l}\right)\left(\operatorname{rank}\left(U^{* l}\right)+1\right)}{2} \leq m
$$

which follows that $\operatorname{rank}\left(U^{* l}\right)<\sqrt{2 m}$. Through the technique used by AMC So et al [14], we construct the following matrices

$$
\begin{aligned}
& U^{* l}=P^{* l}\left(P^{* l}\right)^{T}, \\
& \tilde{X}^{l}=\sum_{j=1}^{n_{2}} \xi^{j l}\left(\xi^{j l}\right)^{T}, \\
& \bar{X}^{l}=P^{* l} \tilde{X}^{l}\left(P^{* l}\right)^{T},
\end{aligned}
$$

where $P^{* l} \in \mathbb{R}^{n_{1} \times r_{l}}$ with $r_{l}=\operatorname{rank}\left(U^{* l}\right)<\sqrt{2 m}$, and $\xi^{j l} \in \mathbb{R}^{r_{l}}$ for $\xi_{i}^{j l}$ are i.i.d. Gaussian random variables with mean 0 and variance $1 / n_{2}$, here $l=1, \cdots, n_{3}, \quad i=1, \cdots, r_{l}$. Clearly $\operatorname{rank}\left(\bar{X}^{l}\right) \leq \operatorname{rank}\left(\tilde{X}^{l}\right) \leq n_{2}$. Notice that $\left(P^{* l}\right)^{T} A^{p} P^{* l} \in \mathbb{R}^{r_{l} \times r_{l}}$, then by proof of Lemma 3.1 [14] and some calculations, we assert that

$$
\left(P^{* l}\right)^{T} A^{p} P^{* l} \bullet \tilde{X}^{l} \leq \alpha\left(m, n_{1}, n_{2}\right) \cdot \operatorname{Tr}\left(P^{* l}\right)^{T} A^{p} P^{* l}=\alpha\left(m, n_{1}, n_{2}\right) \cdot A^{p} \bullet U^{* l}
$$

and

$$
\mathbf{E} A^{0} \bullet \bar{X}^{l}=A^{0} \bullet U^{* l}
$$


with $p=1, \cdots, m$ and $l=1, \cdots, n_{3}$, which means

$$
A^{p} \bullet \bar{X}^{l}=\left(P^{* l}\right)^{T} A^{p} P^{* l} \bullet \tilde{X}^{l} \leq \alpha\left(m, n_{1}, n_{2}\right) \cdot A^{p} \bullet U^{* l} .
$$

Set $X^{l}=\frac{1}{\alpha\left(m, n_{1}, n_{2}\right)} \bar{X}^{l}$, combined with (3.4), easily we get

$$
\begin{aligned}
& X^{l} \succeq 0, \\
& \operatorname{rank}\left(X^{l}\right) \leq \operatorname{rank}\left(\tilde{X}^{l}\right) \leq n_{2}, \\
& \sum_{l} A^{p} \bullet X^{l} \leq \sum_{l} A^{p} \bullet U^{* l} \leq \alpha^{p} \quad \text { for } p=1, \cdots, m .
\end{aligned}
$$

Obviously $X^{l}$ is a feasible solution of problem (3.1). Meanwhile

$$
\begin{aligned}
\mathrm{E} \sum_{l=1}^{n_{3}} A^{0} \bullet X^{l} & =\frac{1}{\alpha\left(m, n_{1}, n_{2}\right)} \sum_{l=1}^{n_{3}} \mathrm{E} A^{0} \bullet \bar{X}^{l} \\
& =\frac{1}{\alpha\left(m, n_{1}, n_{2}\right)} \sum_{l=1}^{n_{3}} A^{0} \bullet U^{* l} \\
& =\frac{1}{\alpha\left(m, n_{1}, n_{2}\right)} v_{\text {mins } d p}^{*} \\
& \leq \frac{1}{\alpha\left(m, n_{1}, n_{2}\right)} v_{\text {minqp }}^{*}
\end{aligned}
$$

where the second equality follows from (3.3). So there exists certain $X^{l}$ such that

$$
v_{\text {minqp }}^{*} \leq \sum_{l=1}^{n_{3}} A^{0} \bullet X^{l} \leq \frac{1}{\alpha\left(m, n_{1}, n_{2}\right)} v_{\text {minqp }}^{*} .
$$

Now we transfer matrix sequence $\left\{X^{l}\right\}_{l=1}^{n_{3}}$ to a third-order tensor $\mathcal{X}^{0}$. Actually the construction process of $\left\{X^{l}\right\}_{l=1}^{n_{3}}$ states that

$$
\begin{aligned}
X^{l} & =\frac{1}{\alpha\left(m, n_{1}, n_{2}\right)} \bar{X}^{l} \\
& =\sum_{j=1}^{n_{2}}\left(\frac{1}{\sqrt{\alpha\left(m, n_{1}, n_{2}\right)}} P^{* l} \xi^{j l}\right)\left(\frac{1}{\sqrt{\alpha\left(m, n_{1}, n_{2}\right)}} P^{* l} \xi^{j l}\right)^{T} \\
& =\sum_{j=1}^{n_{2}} \eta^{j l}\left(\eta^{j l}\right)^{T}
\end{aligned}
$$

here we let $\eta^{j l}=\frac{1}{\sqrt{\alpha\left(m, n_{1}, n_{2}\right)}} P^{* l} \xi^{j l}$ for $j=1, \cdots, n_{2}$ and $l=1, \cdots, n_{3}$.

Set

$$
K^{l}=\left(\eta^{1 l}, \cdots, \eta^{n_{2} l}\right)
$$


and

$$
\mathcal{X}_{i k l}^{0}=K_{i k}^{l},
$$

where $i=1, \cdots, n_{1}, k=1, \cdots, n_{2}$ and $l=1, \cdots, n_{3}$. By the above assumption, we have

$$
\begin{aligned}
\sum_{i j k l} A_{i j}^{0} \mathcal{X}_{i k l}^{0} \mathcal{X}_{j k l}^{0} & =\sum_{i j l} A_{i j}^{0} \sum_{k} K_{i k}^{l} K_{j k}^{l} \\
& =\sum_{i j l} A_{i j}^{0}\left(K^{l}\left(K^{l}\right)^{T}\right)_{i j} \\
& =\sum_{l} A^{0} \bullet\left(K^{l}\left(K^{l}\right)^{T}\right) \\
& =\sum_{l} A^{0} \bullet X^{l}
\end{aligned}
$$

This implies, together with (3.5), that

$$
v_{\text {minqp }}^{*} \leq \sum_{i j k l} A_{i j}^{0} \mathcal{X}_{i k l}^{0} \mathcal{X}_{i k l}^{0} \leq \frac{1}{\alpha\left(m, n_{1}, n_{2}\right)} v_{m i n q p}^{*},
$$

which completes the proof of the theorem.

We remark that the constructive proof of Theorem 3.1 actually provides a polynomial-time algorithm to generate an appropriate feasible solution of the homogeneous QTTP problem (HQ-1), and the approximation ratio is $\Omega\left(\frac{1}{\log m}\right)$ or $\Omega\left(\frac{1}{\sqrt{\log m}}\right)$ which depends on the comparison of $n_{2}$ and $m$.

\section{A class of homogeneous QTTP problems whose SDP relaxations admit no gap with the true optimal values}

For the homogeneous QTTP problem, in this section, after making some assumptions to the coefficient matrices in the objective function and constrains, we can see that its optimal value is the same as the bound obtained by its SDP relaxation and the optimal solution for the original problem can be obtained from the optimal solution of its SDP relaxation.

Consider a homogeneous QTTP problem of the form:

$$
\begin{aligned}
(H Q-2) & \min \sum_{i, j=1}^{n_{1}} \sum_{k=1}^{n_{2}} \sum_{l=1}^{n_{3}} A_{i j}^{0} \mathcal{X}_{i k l} \mathcal{X}_{j k l} \\
\text { s.t. } \quad & \sum_{i, j=1}^{n_{1}} \sum_{k=1}^{n_{2}} \sum_{l=1}^{n_{3}} A_{i j}^{p} \mathcal{X}_{i k l} \mathcal{X}_{j k l} \leq \alpha^{p}, \quad p=1, \cdots, m,
\end{aligned}
$$


where $\alpha^{p} \in \mathbb{R}$ and $A^{0}, A^{p} \in \mathbb{S}^{n_{1}}$ for $p=1, \cdots, m$. Unlike in (HQ-1), here $A^{1}, \cdots, A^{m}$ may be indefinite.

Stretch the three-order tensor $\mathcal{X}$ to $n_{2} n_{3}$ vectors defined by:

$$
x_{i}^{k l}=\mathcal{X}_{i k l}
$$

with $x^{k l} \in \mathbb{R}^{n_{1}}$ for $k=1, \cdots, n_{2}$ and $l=1, \cdots, n_{3}$. By using the correspondence (4.1), we can represent (HQ-2) as the separable indefinite homogeneous quadratic optimization problem:

$$
\begin{array}{ll}
\min & \sum_{k=1}^{n_{2}} \sum_{l=1}^{n_{3}}\left(x^{k l}\right)^{T} A^{0} x^{k l} \\
\text { s.t. } & \sum_{k=1}^{n_{2}} \sum_{l=1}^{n_{3}}\left(x^{k l}\right)^{T} A^{p} x^{k l} \leq \alpha^{p}, \quad p=1, \cdots, m .
\end{array}
$$

The so-called SDP relaxation of (4.2) is

$$
\begin{array}{ll}
\min & \sum_{k l} A^{0} \bullet Y^{k l} \\
\text { s.t. } & \sum_{k l} A^{p} \bullet Y^{k l} \leq \alpha^{p}, \quad p=1, \cdots, m, \\
& Y^{k l} \in \mathbb{S}_{+}^{n_{1}}, \quad k=1, \cdots, n_{2}, l=1, \cdots, n_{3} .
\end{array}
$$

Theorem 4.1

Assume that all off-diagonal elements of $A^{p}(0 \leq p \leq m)$ are nonpositive. Let $Y^{* k l}$ be an optimal solution of the SDP relaxation (4.3) and $x^{* k l}=$ $\left(\sqrt{Y_{11}^{* k l}}, \cdots, \sqrt{Y_{n_{1} n_{1}}^{* k l}}\right)^{T}$ for $1 \leq k \leq n_{2}$ and $1 \leq l \leq n_{3}$. Using transfer strategy (4.1), compress $n_{2} n_{3}$ vectors $\left\{x^{* k l}\right\}_{k l}$ to a third-order tensor $\mathcal{X}^{*}$. Then $\mathcal{X}^{*}$ is an optimal solution of (HQ-2) and $v_{\min }(H Q-2)=v_{\min }(4.3)$.

Proof. By definition, we first observe that

$$
\begin{aligned}
\sum_{k l}\left(x^{* k l}\right)^{T} A^{p} x^{* k l} & =\sum_{k l} \sum_{i j} A_{i j}^{p} x_{i}^{* k l} x_{j}^{* k l} \\
& =\sum_{k l} \sum_{i j} A_{i j}^{p} \sqrt{Y_{i i}^{* k l}} \sqrt{Y_{j j}^{* k l}} \\
& \leq \sum_{k l} \sum_{i j} A_{i j}^{p} Y_{i j}^{* k l} \\
& =\sum_{k l} A^{p} \bullet Y^{* k l}
\end{aligned}
$$


for $p=0,1, \cdots, m$. The above inequality based on the nonpositivity of all offdiagonal elements of $A^{p}(0 \leq p \leq m)$ and the fact that all the $2 \times 2$ principle submatrices of positive semidefinite matrices $\left\{Y^{* k l}\right\}_{k l}$ are nonnegative. Let $p=$ $1, \cdots, m$, combined with the optimality of $\left(Y^{* k l}\right)_{k l}$, (4.4) makes it clear that $\left(x^{* k l}\right)_{k l}$ is a feasible solution of (4.2). Therefore, we obtain that

$$
\sum_{k l} A^{0} \bullet Y^{* k l} \geq \sum_{k l}\left(x^{* k l}\right)^{T} A^{0} x^{* k l} \geq v_{\min }(4.2) \geq v_{\min }(4.3)=\sum_{k l} A^{0} \bullet Y^{* k l} .
$$

Hence $\left(x^{* k l}\right)_{k l}$ is an optimal solution of problem (4.2) which means that $\mathcal{X}^{*}$ is an optimal solution of (HQ-2) and $v_{\min }(H Q-2)=v_{\min }(4.3)$.

The assumption that all off-diagonal elements of $A^{p}(0 \leq p \leq m)$ are nonpositive plays an important role in the proof of Theorem 4.1. This however, does not necessarily require that $A_{i j}^{p}(i \neq j)$, are of the same sign. In fact, $A^{p}(0 \leq$ $p \leq m)$ can be relaxed to be uniformly almost OD-nonpositive. To make this clear, we introduce the following definition.

\section{Definition 4.1}

[7] A family of symmetric matrices $A^{p} \in \mathbb{S}^{n_{1}}(0 \leq p \leq m)$ is said to be uniformly almost OD-nonpositive if there exists a sign vector $\sigma \in\{-1,+1\}^{n_{1}}$ such that

$$
A_{i j}^{p} \sigma_{i} \sigma_{j} \leq 0 \quad \text { for } 1 \leq i<j \leq n_{1}, 0 \leq p \leq m .
$$

As a result, Theorem 4.1 can be extended to the following.

\section{Theorem 4.2}

Assume that the family of symmetric matrices $A^{p}(0 \leq p \leq m)$ is uniformly almost OD-nonpositive with a sign vector $\sigma \in\{-1,+1\}^{n_{1}}$. Let $Y^{* k l}$ be an optimal solution of the following SDP relaxation problem

$$
\begin{array}{ll}
\min & \sum_{k l} \bar{A}^{0} \bullet Y^{k l} \\
\text { s.t. } & \sum_{k l} \bar{A}^{p} \bullet Y^{k l} \leq \alpha^{p}, \quad p=1, \cdots, m, \\
& Y^{k l} \in \mathbb{S}_{+}^{n_{1}}, \quad k=1, \cdots, n_{2}, l=1, \cdots, n_{3}
\end{array}
$$

with $\quad \bar{A}_{i j}^{p}=A_{i j}^{p} \sigma_{i} \sigma_{j} \quad$ for $\quad 1 \leq i, j \leq n_{1} \quad$ and $\quad 1 \leq p \leq m \quad$ and $\quad x^{* k l}=$ $\left(\sigma_{1} \sqrt{Y_{11}^{* k l}}, \cdots, \sigma_{n_{1}} \sqrt{Y_{n_{1} n_{1}}^{* k l}}\right)^{T}$ for $1 \leq k \leq n_{2}$ and $1 \leq l \leq n_{3}$. Using transfer strategy (4.1), compress $n_{2} n_{3}$ vectors $\left\{x^{* k l}\right\}_{k l}$ to a third-order tensor $\mathcal{X}^{*}$. Then $\mathcal{X}^{*}$ is an optimal solution of (HQ-2) and $v_{\min }(H Q-2)=v_{\min }(4.5)$.

Proof. By assumption, we have

$$
A_{i j}^{p} \sigma_{i} \sigma_{j} \leq 0 \quad \text { for } 1 \leq i<j \leq n_{1}, 0 \leq p \leq m
$$


Replace variables $x^{k l}$ by $\sigma \circ x^{k l}$ ( $\circ$ denotes the Hadamard product), it follows that (4.5) is another SDP relaxation of problem (4.2). Set $\left\{x^{* k l}\right\}_{k l}$ as the definition, similar to the proof of Theorem 4.1, we assert the conclusion holds.

At last, we give another tight SDP relaxation result to problem (4.2) when $A^{1}, \cdots, A^{m}$ could be any indefinite matrices. Different from (4.3), the following problem

$$
\begin{array}{ll}
\min & A^{0} \bullet Y \\
\text { s.t. } & A^{p} \bullet Y \leq \alpha^{p}, \quad p=1, \cdots, m, \\
& Y \in \mathbb{S}_{+}^{n_{1}}
\end{array}
$$

is also a SDP relaxation of (4.2). If we get one optimal solution $Y^{*}$ with $\operatorname{rank}\left(Y^{*}\right) \leq n_{2} n_{3}$, then (4.6) is exactly equivalent to problem (4.2). The dual problem to (4.6) is given by

$$
\begin{array}{ll}
\max & -\sum_{p=1}^{m} \alpha^{p} y^{p} \\
\text { s.t. } & Z=A^{0}+\sum_{p=1}^{m} y^{p} A^{p} \succeq 0, \\
& y^{p} \geq 0, \quad p=1, \cdots, m .
\end{array}
$$

\section{Theorem 4.3}

Suppose that SDP relaxation problem (4.6) and its dual (4.7) are solvable. If $m \leq \frac{n_{2} n_{3}\left(n_{2} n_{3}+1\right)}{2}$, then homogeneous tensor programming problem (HQ-2) is solvable, and $v_{\min }(H Q-2)=v_{\min }(4.6)$.

Proof. Similar as the proof of Proposition 2.2, we state that there exists one optimal solution $Y^{*}$ of problem (4.6) which satisfies

$$
\frac{\operatorname{rank}\left(Y^{*}\right)\left(\operatorname{rank}\left(Y^{*}\right)+1\right)}{2} \leq m .
$$

This implies, together with the assumption, that

$$
\operatorname{rank}\left(Y^{*}\right) \leq n_{2} n_{3}
$$

Thus it holds that

$$
v_{\min }(H Q-2)=v_{\min }(4.2)=v_{\min }(4.6),
$$

which completes the proof. 


\section{Acknowledgements}

This work was supported by the National Nature Science Foundation of China (Grant No. 11271206) and Doctoral fund of Chinese Ministry of Education(Grant No. 20120031110024)

\section{REFERENCES}

1. Bader B W, Kolda T G. Algorithm 862: MATLAB tensor classes for fast algorithm prototyping. ACM Trans Math Software, 2006, 32(4): 635- 653

2. Beck A. Quadratic matrix programming. SIAM J Optim, 2007, 17(4): 1224-1238

3. Ben-Tal A, Teboulle M. Hidden convexity in some nonconvex quadratically constrained quadratic programming. Math Program, 1996, 72(1): 51-63

4. Dogan M C, Mendel J. Applications of cumulants to array processing .I. aperture extension and array calibration. IEEE Trans Sig Proc, 1995, 43(5): 1200- 1216

5. Fortin $\mathrm{C}$, Wolkowicz $\mathrm{H}$. The trust region subproblem and semidefinite programming. Optim Methods Softw, 2004, 19(1): 41-67

6. Ghaoui L El, Lebret H. Robust solutions to least-squares problems with uncertain data. SIAM J Matrix Anal Appl, 1997, 18(4): 1035-1064

7. Kim S, Kojima M. Exact solutions of some nonconvex quadratic optimization problems via SDP and SOCP relaxations. Comput Optim Appl, 2003, 26(2): 143-154

8. Kolda T G, Bader B W. Tensor Decompositions and Applications. SIAM Review, 2009, 51(3): 455- 500

9. Lathauwer L de, Castaing J. Tensor-based techniques for the blind separation of ds-cdma signals. Signal Processing, 2007, 87(2): 322- 336

10. Luo Z Q, Sidiropoulos N D, Tseng P, Zhang S Z. Approximation Bounds for Quadratic Optimization with Homogeneous Quadratic Constraints. SIAM J Optim, 2007, 18(1): 1-28

11. Pakati G. On the rank of extreme matrices in semidefinite programs and the multiplicity of optimal eigenvalues. Math Oper Res, 1998, 23(2): 339-358

12. Polyak B T. Convexity of quadratic transformations and its use in control and optimization. $\mathrm{J}$ Optim Theory Appl, 1998, 99(3): 553-583

13. Sidiropoulos N D, Giannakis G B, Bro R. Blind PARAFAC receivers for DS-CDMA systems. IEEE Trans on Sig Proc, 2000, 48(3): 810- 823

14. So A M-C, Ye Y Y, Zhang J W. A unified theorem on SDP rank reduction. Math Oper Res, 2008, 33(4): 910-920

15. Stern R J, Wolkowicz H. Indefinite trust region subproblems and nonsymmetric eigenvalue perturbations. SIAM J Optim, 1995, 5(2): 286-313

16. Swami A, Giannakis G, Shamsunder S. Multichannel ARMA processes. IEEE Trans Sig Proc, 1994, 42(4): 898- 913

17. Ye Y Y, Zhang S Z. New results on quadratic minimization. SIAM J Optim, 2003, 14(1): 245-267 